\title{
TEMPERATURES OF LINEARLY AND NONLINEARLY RADIATING SEMI-INFINITE RODS*
}

\author{
$\mathrm{By}$ \\ C. Y. CHAN \\ Florida State University
}

1. Introduction. Let $D=\{(x, t): x>0, t>0\}, S=\{(0, t): t>0\}$, and $C=$ $\{(x, 0): x \geq 0\}$ with $D^{-}$and $S^{-}$denoting the closures of $D$ and $S$ respectively. Also let $u(x, t)$ be the temperature distribution in a homogeneous, isotropic and semi-infinite rod, which is radiating heat along its entire length at the rates proportional to $u$ and $g(x, t ; u)$, and also at the end $x=0$ at the rates proportional to $u$ and $B(t ; u)$. Without loss of generality in the main results, we assume the diffusivity of the rod to be one. If energy is supplied to the end $x=0$ at a rate proportional to some function $f(t)$, and the initial distribution of temperature is given by $\phi(x)$, then $u(x, t)$ is determined by the following initial boundary value problem:

$$
\begin{gathered}
L u \equiv u_{x x}(x, t)-h u(x, t)-u_{t}(x, t)=k g(x, t ; u) \text { in } D, \\
u(x, 0)=\phi(x) \quad \text { on } C \\
A u \equiv u_{x}(0, t)-b u(0, t)=a B(t ; u)-f(t) \quad \text { on } S,
\end{gathered}
$$

where $u$ is assumed to tend to zero as $x$ tends to infinity for $t \geq 0$. Here $h, k, b$ and $a$ are given constants with $h, k$ and $a$ being nonnegative while the given functions $g, \phi, B$ and $f$ are piecewise continuous with $\phi$ and $f$ being bounded and nonnegative, and $f \equiv 0$ for $T<t<\infty$, where $T$ is a nonnegative constant.

When $k=\phi=0$, and $B(t ; u)=u^{n}(0, t)$, where $n$ is a positive constant, our problem (1.1)-(1.3) reduces to the one studied recently by Hartka [2]. If in addition $h=b=0$, then we have the problem considered by Keller and Olmstead [3]. For further references, we refer to these papers, where existence of the nonnegative solution for each problem was established by constructing the surface temperature $u(0, t)$. On the other hand, if $B(t ; u)=u^{n}(0, t)$, then $n=1$ corresponds to the Newton law of cooling, and $n=4$ corresponds to the Stefan radiation law for black bodies.

The main purpose of this paper is to establish existence of the maximal and the minimal nonnegative solutions of the problem (1.1)-(1.3), to give conditions under which these coincide, and to construct upper and lower bounds for the nonnegative solutions. Our quest for nonnegative solutions is motivated by the physical concept of the absolute temperature. The methods used here are different from those in the above-mentioned papers.

Instead of treating the special case $B(t ; u)=u^{n}(0, t)$, we shall require $B$ to satisfy some or all of the following conditions:

*Received September 20, 1974; revised version received February 20, 1975. 
$\left(B_{1}\right)$ there exists a bounded, nonnegative and piecewise continuous function $q(x, t)$ such that for bounded functions $u(x, t)$ and $v(x, t)$ in $D^{-}, B(t ; u(0, t))-B(t ; v(0, t)) \leq$ $q[u(0, t)-v(0, t)]$ if $u>v$ at $(0, t)$;

$\left(B_{2}\right) B(t ; 0)=0$;

$\left(B_{3}\right) u>0$ at the point $(0, t)$ implies $B(t ; u(0, t)) \geq 0$;

$\left(B_{4}\right) u>v$ at the point $(0, t)$ implies $B(t ; u(0, t)) \geq B(t ; v(0, t))$.

Also we shall need the function $g$ to satisfy some or all of the following assumptions:

$\left(g_{1}\right)$ there exists a bounded, nonnegative and piecewise continuous function $p(x, t)$ such that for bounded functions $u(x, t)$ and $v(x, t)$ in $D^{-}, g(x, t ; u(x, t))-g(x, t ; v(x, t)) \leq$ $p[u(x, t)-v(x, t)]$ if $u>v$ at $(x, t)$;

$\left(g_{2}\right) \quad g(x, t ; 0)=0$

$\left(g_{3}\right) u>0$ at the point $(x, t)$ implies $g(x, t ; u(x, t)) \geq 0$;

$\left(g_{4}\right) u>v$ at the point $(x, t)$ implies $g(x, t ; u(x, t)) \geq g(x, t ; v(x, t))$.

Here we note in particular that $q$ and $p$ in conditions $\left(B_{1}\right)$ and $\left(g_{1}\right)$ respectively can be replaced by appropriate nonnegative constants. Also, conditions $\left(B_{2}\right)$ and $\left(B_{4}\right)$ taken together imply condition $\left(B_{3}\right)$ while assumption $\left(g_{3}\right)$ follows from assumptions $\left(g_{2}\right)$ and $\left(g_{4}\right)$.

Let

$$
K(x, t ; \xi, \tau)=\left\{2^{-1}[\pi(t-\tau)]^{-1 / 2}\right\} \exp \left\{-(x-\xi)^{2} /[4(t-\tau)]\right\} .
$$

Also let $Z(x, t ; \xi, \tau)$ be the solution of $l Z \equiv Z_{x x}(x, t ; \xi, \tau)-\lambda Z(x, t ; \xi, \tau)-Z_{t}(x, t ; \xi, \tau)=0$ for $x, \xi>0, t>\tau$,

$$
\begin{gathered}
Z(x, \tau ; \xi, \tau)=\delta(x-\xi), \\
\gamma Z \equiv Z_{x}(0, t ; \xi, \tau)-\theta Z(0, t ; \xi, \tau)=0,
\end{gathered}
$$

where $\delta$ is the Dirac distribution, $\lambda$ and $\theta$ are given constants. The function $Z$ is a fundamental solution called the Neumann function (cf. Chan [1]) of $l w(x, t)=0$ in $D$ and $\gamma w(0, t)=0$ on $S$. Let

$$
Z(x, t ; \xi, \tau)=z(x, t ; \xi, \tau) \exp [-\lambda(t-\tau)] .
$$

It follows from Stakgold [5, pp. 209-210] that

$$
\begin{aligned}
z(x, t ; \xi, \tau)= & K(x, t ; \xi, \tau)+K(-x, t ; \xi, \tau) \\
& -\theta[\pi(t-\tau)]^{-1 / 2} \int_{0}^{\infty} \exp \left\{-\theta y-(x+\xi+y)^{2} /[4(t-\tau)]\right\} d y .
\end{aligned}
$$

We shall need the following two positivity lemmas.

Lemma 1. $Z(x, t ; \xi, \tau)>0$ for $x, \xi \geq 0, t>\tau \geq 0$.

Proof. If $\theta=0$, then it follows from (1.4) and (1.5) that the lemma is proved. If $\theta \neq 0$, then by integrating the third term on the right-hand side of (1.5) by parts, we have

$$
\begin{aligned}
z(x, t ; \xi, \tau)=K(x, t ; \xi, \tau) & -K(-x, t ; \xi, \tau) \\
& +(t-\tau)^{-1} \int_{0}^{\infty}[\exp (-\theta y)](x+\xi+y) K(-x, t ; \xi+y, \tau) d y,
\end{aligned}
$$

from which the Lemma follows with the use of (1.4). 
The solution $w(x, t)$ of the problem

$$
\begin{aligned}
& l w=G(x, t) \text { in } D, \\
& w(x, 0)=\Phi(x) \quad \text { on } C \text {, } \\
& \gamma w=F(t) \quad \text { on } S,
\end{aligned}
$$

where $w$ tends to zero as $x$ tends to infinity for $t \geq 0$, is given by

$$
\begin{aligned}
w(x, t)=\int_{0}^{\infty} Z(x, t ; \xi, 0) \Phi(\xi) d \xi-\int_{0}^{t} \int_{0}^{\infty} Z(x, t ; \xi, \tau) G(\xi, \tau) d \xi d \tau & \\
& -\int_{0}^{t} Z(x, t ; 0, \tau) F(\tau) d \tau .
\end{aligned}
$$

This together with Lemma 1 gives the following result.

Lemma 2. If in (1.6)-(1.8), $G \leq 0, \Phi \geq 0$, and $F \leq 0$, then $w \geq 0$ in $D^{-}$.

In Sec. 2, we establish existence of the maximal and the minimal nonnegative solutions, which are constructed respectively as the limits of a monotone nonincreasing sequence of upper bounds and a monotone nondecreasing sequence of lower bounds. Hence the error involved in using a certain approximate solution can be estimated. Also we give conditions which ensure uniqueness of the solution. With uniqueness, the rate of convergence for each of the above sequences is shown to be geometrical. In Sec. 3, we show that an iteration scheme of the Picard type gives an alternating sequence consisting of two monotone subsequences bounding any nonnegative solution from above and below. Thus each successive iteration gives a more accurate pointwise upper or lower bound. Under additional assumptions, this sequence is shown to converge uniformly and geometrically to a solution.

2. Maximal and minimal solutions. Since we are interested in nonnegative solutions here, we shall use the following definition.

Definition. A solution $M(x, t)(m(x, t))$ of the problem (1.1)-(1.3) is said to be maximal (minimal) if $u(x, t) \leq M(x, t)(m(x, t) \leq u(x, t))$ for any nonnegative solution $u(x, t)$.

Let us construct a sequence $\left\{M_{i}(x, t)\right\}$ by $L M_{0}=0$ in $D, M_{0}=\phi$ on $C, A M_{0}=-f$ on $S$, and for $i=0,1,2, \cdots$,

$$
\begin{aligned}
L M_{i+1} & =k g\left(x, t ; M_{i}\right)+r\left(M_{i+1}-M_{i}\right) \text { in } D, \\
M_{i+1} & =\phi \text { on } C, \\
A M_{i+1} & =a B\left(t ; M_{i}\right)+s\left(M_{i+1}-M_{i}\right)-f \text { on } S,
\end{aligned}
$$

where $M_{i}$ tends to zero as $x$ tends to infinity for $t \geq 0, r$ and $s$ are constants chosen to be $r \geq k p$ and $s \geq a q$ with $p$ and $q$ being given in conditions $\left(g_{1}\right)$ and $\left(B_{1}\right)$ respectively. The following theorem shows that this constructed sequence forms a uniformly bounded and monotone nonincreasing sequence of upper bounds for solutions of the problem (1.1)-(1.3), and also establishes existence of the maximal solution.

Theorem 1. Under assumptions $\left(g_{1}\right)-\left(g_{3}\right)$ and $\left(B_{1}\right)-\left(B_{3}\right)$, and $b^{2}-h \leq 0$ if $b<0$, the sequence $\left\{M_{i}\right\}$ satisfies the inequalities

$$
0 \leq M_{i+1} \leq M_{i} \leq c_{1} \text { in } D^{-}, \quad i=0,1,2, \cdots
$$


where $c_{1}$ is a nonnegative constant; furthermore, it converges to the maximal solution of the problem (1.1)-(1.3).

Proof. By Lemma 2, $M_{0} \geq 0$ in $D^{-}$. On the other hand,

$$
M_{0}(x, t)=\int_{0}^{\infty} R(x, t ; \xi, 0) \phi(\xi) d \xi+\int_{0}^{t} R(x, t ; 0, \tau) f(\tau) d \tau,
$$

where $R$ is the Neumann function of $L w=0$ in $D$ and $A w=0$ on $S$. From (1.4) and Stakgold [5, pp. 209-210], $R$ can be written in the form

$$
\begin{aligned}
R(x, t ; \xi, \tau)=\{\exp [-h(t-\tau)]\}[ & K(x, t ; \xi, \tau)+K(-x, t ; \xi, \tau)] \\
- & b\left\{\exp \left[b(x+\xi)+\left(b^{2}-h\right)(t-\tau)\right]\right\} \\
& \cdot\left\{1-\operatorname{erf}\left[(x+\xi) /\left(2(t-\tau)^{1 / 2}\right)+b(t-\tau)^{1 / 2}\right]\right\},
\end{aligned}
$$

where $\operatorname{erf} y=2 \pi^{-1 / 2} \int_{0}{ }^{y} \exp \left(-\xi^{2}\right) d \xi$ is the error function. Since $\phi$ and $f$ are bounded, let $c_{2}$ and $c_{3}$ be nonnegative constants such that $\phi \leq c_{2}$ and $f \leq c_{3}$. Also, let

$$
\begin{aligned}
E & =t^{1 / 2} \quad \text { if } \quad t \leq T, \\
& =t^{1 / 2}-(t-T)^{1 / 2} \quad \text { if } \quad t>T, \\
F & =\left(h-b^{2}\right)^{-1}\left\{\exp \left[T\left(h-b^{2}\right)\right]-1\right\} \quad \text { if } \quad b<0 \quad \text { and } \quad b^{2}-h<0, \\
& =T \quad \text { if } \quad b<0 \text { and } b^{2}-h=0 .
\end{aligned}
$$

Using $f \equiv 0$ for $t>T$, and the facts that $0 \leq \operatorname{erf} y \leq 1$ and $e^{-\nu} \leq 1$ for $y \geq 0$, we obtain $M_{0}(x, t) \leq[\exp (-h t)]\left[3 c_{2} / 2+2 c_{3} E \pi^{-1 / 2} \exp (h T)\right]$ if $b \geq 0$, $M_{0}(x, t) \leq[\exp (-h t)]\left\{c_{2}\left[3 / 2+2 \exp \left(b^{2} t\right)\right]\right.$

$$
\left.+2 c_{3}\left[\pi^{-1 / 2} E \exp (h T)+|b| F \exp \left(b^{2} t\right)\right]\right\} \quad \text { if } \quad b<0 .
$$

Since $b^{2}-h \leq 0$ if $b<0$, we have

$$
M_{0}(x, t) \leq 7 c_{2} / 2+2 c_{3}\left[\pi^{-1 / 2} T^{1 / 2} \exp (h T)+|b| F\right],
$$

irrespective of the sign of $b$. Denoting the right-hand side hy $c_{1}$, we have $M_{0}(x, t) \leq c_{1}$.

From assumptions $\left(g_{1}\right)$ and $\left(g_{2}\right)$, we have

$$
g\left(x, t ; M_{0}\right)-p M_{0}(x, t) \leq g(x, t ; 0)=0 .
$$

Similarly, from assumptions $\left(B_{1}\right)$ and $\left(B_{2}\right)$, we have

$$
B\left(t ; M_{0}\right)-q M_{0}(0, t) \leq B(t ; 0)=0 .
$$

It follows from (2.1) and (2.3) respectively that

$$
\begin{aligned}
& (L-r) M_{1} \leq k\left[g\left(x, t ; M_{0}\right)-p M_{0}\right] \leq 0 \text { in } \quad D, \\
& (A-s) M_{1} \leq a\left[B\left(t ; M_{0}\right)-q M_{0}\right]-f \leq 0 \text { on } S .
\end{aligned}
$$

Since $\phi \geq 0$, it follows from Lemma 2 that $M_{1} \geq 0$ in $D^{-}$.

Next, we show that $M_{0} \geq M_{1}$ in $D^{-}$. Since $M_{0} \geq 0$ in $D^{-}$, it follows from assumptions $\left(g_{2}\right),\left(g_{3}\right),\left(B_{2}\right)$ and $\left(B_{3}\right)$ that $g\left(x, t ; M_{0}\right) \geq 0$ and $B\left(t ; M_{0}\right) \geq 0$. Thus,

$$
\begin{aligned}
& (L-r)\left(M_{0}-M_{1}\right)=-k g\left(x, t ; M_{0}\right) \leq 0 \text { in } D, \\
& (A-s)\left(M_{0}-M_{1}\right)=-a B\left(t ; M_{0}\right) \leq 0 \text { on } S .
\end{aligned}
$$


Since $M_{0}-M_{1}=0$ on $C$, we have $M_{0} \geq M_{1}$ in $D^{-}$.

To establish (2.4), let us assume that for a particular value of $i$ (say $j \geq 1$ ),

$$
0 \leq M_{i} \leq M_{i-1} \leq \cdots \leq M_{0} \leq c_{1} \text { in } D^{-} .
$$

Then it follows from assumptions $\left(g_{1}\right)$ and $\left(g_{2}\right)$ that

$$
g\left(x, t ; M_{i}\right) \leq p M_{i},
$$

and from assumptions $\left(B_{1}\right)$ and $\left(B_{2}\right)$ that

$$
B\left(t ; M_{i}\right) \leq q M_{i} .
$$

Thus,

$$
\begin{aligned}
& (L-r) M_{i+1} \leq(k p-r) M_{i} \leq 0 \quad \text { in } \quad D, \\
& (A-s) M_{i+1} \leq(a q-s) M_{i}-f \leq 0 \quad \text { on } \quad S .
\end{aligned}
$$

Hence, $M_{i+1} \geq 0$ in $D^{-}$. Using assumptions $\left(g_{1}\right)$ and $\left(B_{1}\right)$, we have

$$
\begin{aligned}
& (L-r)\left(M_{i}-M_{i+1}\right) \leq(k p-r)\left(M_{i-1}-M_{i}\right) \leq 0 \quad \text { in } \quad D, \\
& (A-s)\left(M_{i}-M_{i+1}\right) \leq(a q-s)\left(M_{i-1}-M_{j}\right) \leq 0 \text { on } S .
\end{aligned}
$$

Since $M_{i}-M_{i+1}=0$ on $C$, it follows that $M_{i} \geq M_{i+1}$ in $D^{-}$. From the principle of mathematical induction, we have (2.4).

Since the sequence $\left\{M_{i}\right\}$ is monotone nonincreasing and uniformly bounded, there exists a function $M$ to which the sequence converges pointwise. To show that $M$ is a solution of the problem (1.1)-(1.3), let us rewrite the iteration scheme (2.1)-(2.3) equivalently as

$$
\begin{aligned}
& M_{i+1}(x, t)=\int_{0}^{\infty} R(x, t ; \xi, 0) \phi(\xi) d \xi \\
&-\int_{0}^{t} \int_{0}^{\infty} R(x, t ; \xi, \tau)\left[k g\left(\xi, \tau ; M_{i}\right)+r\left(M_{i+1}-M_{i}\right)\right] d \xi d \tau \\
&-\int_{0}^{t} R(x, t ; 0, \tau)\left[a B\left(\tau ; M_{i}\right)+s\left(M_{i+1}-M_{i}\right)-f(\tau)\right] d \tau
\end{aligned}
$$

By Lemma 1, (2.4), (2.7) and (2.8), the integrands in the second and the third integrals on the right-hand side of (2.9) are bounded respectively by

$$
\begin{gathered}
(k p+r) M_{0}(\xi, \tau) R(x, t ; \xi, \tau), \\
{\left[a q M_{0}(0, \tau)+s M_{0}(0, \tau)+c_{3}\right] R(x, t ; 0, \tau),}
\end{gathered}
$$

both of which are integrable over their respective regions of integration. As $i$ tends to infinity in (2.9), it follows from the Lebesgue convergence theorem (ef. Royden [4, p. 200]) that we can interchange the limit and the integration processes. Hence,

$$
\begin{aligned}
& M(x, t)=\int_{0}^{\infty} R(x, t ; \xi, 0) \phi(\xi) d \xi-k \int_{0}^{t} \int_{0}^{\infty} R(x, t ; \xi, \tau) g(\xi, \tau ; M I) d \xi d \tau \\
& \quad-\int_{0}^{t} R(x, t ; 0, \tau)[a B(\tau ; M I)-f(\tau)] d \tau .
\end{aligned}
$$

This implies that $M$ is a solution of the problem (1.1)-(1.3). 
To show that $M$ is maximal, let $u$ be any nonnegative solution of the problem (1.1)-(1.3). Then,

$$
\begin{aligned}
& L\left(M_{0}-u\right)=-k g(r, t ; u) \leq 0 \text { in } D, \\
& A\left(I_{0}-u\right)=-a B(t ; u) \leq 0 \text { on } S .
\end{aligned}
$$

As $M_{0}-u=0$ on $C$, we have $M_{0} \geq u$ in $D^{-}$. Let us assume that $u \leq M_{j}$ in $D^{-}$for some $j$. Using assumptions $\left(g_{1}\right)$ and $\left(B_{1}\right)$, we have

$$
\begin{aligned}
& (L-r)\left(M_{i+1}-u\right) \leq(k p-r)\left(M_{i}-u\right) \leq 0 \quad \text { in } \quad D, \\
& (A-s)\left(M_{i+1}-u\right) \leq(a q-s)\left(M_{i}-u\right) \leq 0 \quad \text { on } \quad S .
\end{aligned}
$$

Since $M_{i+1}-u=0$ on $C$, it follows that $M_{j+1} \geq u$ in $D^{-}$. From the principle of mathematical induction, we have $u \leq M_{i}$ in $D^{-}$for $i=0,1,2, \cdots$. Hence $u \leq M$ in $D^{-}$. This shows that $M$ is the maximal solution.

If $b^{2}-h<0$, then it follows from (2.6) that $M_{0}(x, t)$ tends to zero as $t$ tends to infinity; this is physically obvious since the temperature must approach zero if heat is lost along the length of the rod faster than it can be absorbed at the end (ef. Hartka [2]).

Our next theorem gives a monotone nondecreasing sequence of lower bounds for nonnegative solutions of the problem (1.1)-(1.3), and also existence of the minimal solution. We omit its proof here since it is similar to that of Theorem 1 with some obvious modifications.

Theorem 2. Under the hypotheses of Theorem 1, the sequence $\left\{m_{i}(x, t)\right\}$ constructed by $m_{0} \equiv 0$ in $D^{-}$, and for $i=0,1,2, \cdots$,

$$
\begin{aligned}
L m_{i+1} & =l i g\left(r, t ; m_{i}\right)+r\left(m_{i+1}-m_{i}\right) \text { in } D, \\
m_{i+1} & =\phi \text { on } C, \\
A m_{i+1} & =a B\left(t ; m_{i}\right)+s\left(m_{i+1}-m_{i}\right)-f \text { on } s,
\end{aligned}
$$

where $m_{i}$ tends to zero as $x$ tends to infinity for $t \geq 0$, satisfies

$$
0 \leq m_{i} \leq m_{i+1} \leq M_{0} \leq c_{1} \text { in } D^{-}, \quad i=0,1,2, \cdots,
$$

and converges to the minimal solution $m$ of the problem (1.1)-(1.3).

That the maximal solution is nonnegative follows from its definition. Because of (2.10), the minimal solution is also nonnegative. The following result gives the conditions under which we have a unique solution.

Theorem 3. Under the hypotheses of Theorem 1 and the assumptions $\left(g_{4}\right)$ and $\left(B_{4}\right)$, there exists a unique nonnegative solution of the problem (1.1)-(1.3).

Proof. By Theorems 1 and 2, we have existence of the maximal solution $M$ and the minimal solution $m$. Thus,

$$
\begin{aligned}
0 \leq M(x, t)-m(x, t) & =-k \int_{0}^{t} \int_{0}^{\infty} R(x, t ; \xi, \tau)[g(\xi, \tau ; M)-g(\xi, \tau ; m)] d \xi d \tau \\
& -a \int_{0}^{t} R(x, t ; 0, \tau)[B(\tau ; M)-B(\tau ; m)] d \tau \text { in } D^{-}
\end{aligned}
$$

It follows from Lemma 1 and assumptions $\left(g_{4}\right)$ and $\left(B_{4}\right)$ that the integrands in the last 
two integrals are nonnegative, and hence from (2.11) we have

$$
0 \leq M(x, t)-m(x, t) \leq 0 \text { in } D^{-} .
$$

Thus, $M \equiv m$ in $D^{-}$, and hence the solution is unique.

Our next result gives the rate of convergence of each of the sequences $\left\{M_{i}\right\}$ and $\left\{m_{i}\right\}$ to the solution.

Theorem 4. Under the hypotheses of Theorem 3, each of the sequences $\left\{M_{i}\right\}$ and $\left\{m_{i}\right\}$ converges uniformly and geometrically to the unique nonnegative solution

$$
u=\lim _{i \rightarrow \infty} M_{i}=\lim _{i \rightarrow \infty} m_{i}
$$

of the problem (1.1)-(1.3).

Proof. Since $M_{i+1} \leq M_{i}$ in $D^{-}, k \geq 0$, and $a \geq 0$, it follows from (2.9), Lemma 1, and assumptions $\left(B_{4}\right)$ and $\left(g_{4}\right)$ that

$$
\begin{aligned}
M_{i}(x, t)-M_{i+1}(x, t) \leq r \int_{0}^{t} \int_{0}^{\infty} R(x, t ; \xi, \tau)\left(M_{i-1}\right. & \left.-M_{i}\right) d \xi d \tau \\
& \quad+s \int_{0}^{t} R(x, t ; 0, \tau)\left(M_{i-1}-M_{i}\right) d \tau
\end{aligned}
$$

after having dropped out the nonpositive terms. Let

$$
\rho_{i}=\sup _{(x, t) \in D^{-}}\left|M_{i+1}-M_{i}\right|
$$

and $c_{4}=\max \{r, s\}$. Then

$$
\rho_{i} \leq c_{4} \rho_{i-1}\left[\int_{0}^{t} \int_{0}^{\infty} R(x, t ; \xi, \tau) d \xi d \tau+\int_{0}^{t} R(x, t ; 0, \tau) d \tau\right] .
$$

Using (2.5), and the facts that $0 \leq \operatorname{erf} y \leq 1$ and $e^{-y} \leq 1$ for $y \geq 0$, we have

$$
\rho_{i} \leq c_{4}\left[(7 / 2+2|b|) t+2 \pi^{-1 / 2} t^{1 / 2}\right] \rho_{i-1} .
$$

Let the quantity inside the square brackets be denoted by $\mu(t)$, which is nonnegative. From (2.4), $\rho_{0} \leq c_{1}$. Thus it follows from induction that

$$
\rho_{n} \leq\left[c_{4} \mu(t)\right]^{n} \rho_{0} \leq c_{1}\left[c_{4} \mu(t)\right]^{n} .
$$

Let us choose the time interval $[0, \sigma]$ such that $c_{4} \mu(t)<1$ so that the sequence $\left\{M_{i}\right\}$ converges uniformly and geometrically to a solution on $[0, \sigma]$ with the use of the Lebesgue convergence theorem. Since $M_{i} \geq 0$, this solution is nonnegative.

Next, we start from $t=\sigma-\eta$, where $\eta$ is an arbitrarily chosen positive constant such that $\sigma-\eta>0$. Using an argument similar to the above, we obtain the inequality

$$
c_{4}\left[(7 / 2+2|b|)(t-\sigma+\eta)+2 \pi^{-1 / 2}(t-\sigma+\eta)^{1 / 2}\right]<1
$$

restricting the time interval for convergence. To satisfy this inequality, we can choose the time interval to be $[\sigma-\eta, 2 \sigma-\eta]$. Thus, $\left\{M_{i}\right\}$ converges uniformly and geometrically to a nonnegative solution for $0 \leq t \leq 2 \sigma-\eta$. By repeating the above procedures, we have uniform and geometrical convergence of $\left\{M_{i}\right\}$ to a nonnegative solution of the problem (1.1)-(1.3).

A similar argument applied to the sequence $\left\{m_{i}\right\}$ shows that $\left\{m_{i}\right\}$ converges uniformly 
and geometrically to a nonnegative solution. From Theorem 3 , the nonnegative solution of the problem (1.1)-(1.3) is unique. Thus, (2.12) holds.

3. Alternating bounds. Let us construct the sequence $\left\{u_{i}(x, t)\right\}$ by $u_{0} \equiv M_{0}$ in $D^{-}$, and for $i=0,1,2, \cdots$,

$$
\begin{aligned}
L u_{i+1} & =k g\left(x, t ; u_{i}\right) \text { in } D, \\
u_{i+1} & =\phi \text { on } C, \\
A u_{i ! 1} & =a B\left(t ; u_{i}\right)-f \text { on } S,
\end{aligned}
$$

where $u_{i}$ tends to zero as $x$ tends to infinity for $t \geq 0$. This gives an alternating sequence, which is different from that by Keller and Olmstead [3].

Theorem 5. If assumptions $\left(g_{2}\right),\left(g_{4}\right),\left(B_{2}\right)$ and $\left(B_{4}\right)$ hold, then any nonnegative solution $u$ of the problem (1.1)-(1.3) satisfies

$$
u_{1} \leq \cdots \leq u_{2 i+1} \leq \cdots \leq u \leq \cdots \leq u_{2 i} \leq \cdots \leq u_{0} \text { in } D^{-} .
$$

Proof. First, we show that $u \leq u_{0}$ in $D^{-}$. Since assumptions $\left(g_{2}\right)$ and $\left(g_{4}\right)$ imply $\left(g_{3}\right)$, and $u \geq 0$ in $D^{-}$, we have $g(x, t ; u) \geq 0$ in $D$. Similarly, assumptions $\left(B_{2}\right)$ and $\left(B_{4}\right)$ imply $\left(B_{3}\right)$, and we have $B(t ; u) \geq 0$ on $S$. Thus,

$$
\begin{aligned}
& L\left(u_{0}-u\right)=-k g(x, t ; u) \leq 0 \text { in } D, \\
& A\left(u_{0}-u\right)=-a B(t ; u) \leq 0 \text { on } S .
\end{aligned}
$$

Since $u_{0}-u=0$ on $C$, we have $u_{0} \geq u$ in $D^{-}$by using Lemma 2 .

Because $u \leq u_{0}$ in $D^{-}$, it follows from assumptions $\left(g_{4}\right)$ and $\left(B_{4}\right)$ respectively that $L\left(u-u_{1}\right) \leq 0$ in $D$, and $A\left(u-u_{1}\right) \leq 0$ on $S$. As $u-u_{1}=0$ on $C$, we have $u \geq u_{1}$ in $D^{-}$.

Let us assume that for a particular value of $i$, say $j$,

$$
u_{1} \leq \cdots \leq u_{2 j+1} \leq u \leq u_{2 i} \leq \cdots \leq u_{0} \text { in } D^{-} .
$$

Then for $i=j+1$, it follows from assumptions $\left(g_{4}\right)$ and $\left(B_{4}\right)$ respectively that $L\left(u_{2 i+2}-u\right) \leq 0$ in $D$, and $A\left(u_{2 j+2}-u\right) \leq 0$ on $S$. Since $u_{2 i+2}-u=0$ on $C$, we have $u_{2 i+2} \geq u$ in $D^{-}$. By repeating the argument for $u_{2 j}-u_{2 j+2}, u-u_{2 j+3}$, and $u_{2 j+3}-u_{2 j+1}$ respectively, we have $u_{2 j} \geq u_{2 j+2}, u \geq u_{2 j+3}$, and $u_{2 j+3} \geq u_{2 j+1}$, From the principle of mathematical induction, we have (3.1).

The subsequence $\left\{u_{2 i+1}\right\}$ is monotone nondecreasing and bounded above by $u_{0}$ while the subsequence $\left\{u_{2 i}\right\}$ is monotone nonincreasing and bounded below by $u_{1}$. It has not been proved above that the alternating sequence converges to a solution of the problem (1.1)-(1.3). Even if the odd and the even subsequences converge respectively to a lower bound and an upper bound, neither of them may be a solution. Our next result shows that under additional conditions, the sequence $\left\{u_{i}\right\}$ does converge to a solution, which need not be nonnegative.

Theorem 6. Under the hypotheses of Theorem 5, assumptions $\left(g_{1}\right)$ and $\left(B_{1}\right)$, $b^{2}-h \leq 0$ if $b<0$, and $g \equiv B \equiv 0$ for $t>c$ where $c$ is a nonnegative constant, the sequence $\left\{u_{i}\right\}$ converges uniformly and geometrically to a solution of the problem (1.1)-(1.3).

Proof. First, let us show that $u_{1}$ is bounded bclow by a constant. From (2.4), 
$0 \leq u_{0} \leq c_{1}$ in $D^{-}$. Thus, $g\left(x, t ; u_{0}\right) \geq 0$ and $B\left(t ; u_{0}\right) \geq 0$. It follows that

$$
u_{1}(x, t) \geq-k \int_{0}^{t} \int_{0}^{\infty} R(x, t ; \xi, \tau) g\left(\xi, \tau ; u_{0}\right) d \xi d \tau-a \int_{0}^{t} R(x, t ; 0, \tau) B\left(\tau ; u_{0}\right) d \tau
$$

after having dropped out the nonnegative terms on the right hand side. Let

$$
\begin{aligned}
d=t & \text { if } t \leq c \\
=c & \text { if } t>c .
\end{aligned}
$$

Using assumptions $\left(g_{1}\right),\left(g_{2}\right),\left(B_{1}\right)$ and $\left(B_{2}\right), r \geq k p, s \geq a q$, and $c_{4}=\max \{r, s\}$, we have

$$
u_{1}(x, t) \geq-c_{1} c_{4}\left[\int_{0}^{d} \int_{0}^{\infty} R(x, t ; \xi, \tau) d \xi d \tau+\int_{0}^{d} R(x, t ; 0, \tau) d \tau\right] .
$$

A reasoning analogous to that in arriving at (2.14) from (2.13) gives

$$
u_{1}(x, l) \geq-c_{1} c_{4}\left\{(7 / 2+2|b|) d+2 \pi^{-1 / 2}\left[t^{1 / 2}-(t-d)^{1 / 2}\right]\right\} .
$$

Since

$$
t^{1 / 2}-(t-d)^{1 / 2} \leq c^{1 / 2}
$$

we have

$$
u_{1}(x, t) \geq-c_{1} c_{4}\left[(7 / 2+2|b|) c+2 \pi^{-1 / 2} c^{1 / 2}\right] .
$$

Thus, $u_{1}$ is bounded below. An argument similar to that in proving the convergence of $\left\{M_{1}\right\}$ in Theorem 4 shows that the sequence $\left\{u_{i}\right\}$ converges uniformly and geometrically to a solution of the problem (1.1)-(1.3).

We note that in each step of the constructions of the maximal and the minimal solutions, the same Neumann function of $(L-r) w=0$ in $D$ and $(A-s) w=0$ on $S$ is used, except in the initial step of constructing $M_{0}$ when we use the Neumann function of $L w=0$ in $D$ and $A w=0$ on $S$. This latter function is also used in the constructions of the alternating bounds.

\section{REFERENCES}

[1] C. Y. Chan, Positive solutions for nonlinear parabolic second initial boundary value problems, Quart. Appl. Math. 31, 443-454 (1974)

[2] J. E. Hartka, Temperature of a semi-infinite rod which radiates both linearly and nonlinearly, Quart. Appl. Math. 32, 101-111 (1974)

[3] J. B. Keller and W. E. Olmstead, Temperature of a nonlinearly radiating semi-infinite solid, Quart. Appl. Math. 29, 559-566 (1972)

[4] H. L. Royden, Real analysis, Macmillan Co., New York, 1963, p. 200

[5] I. Stakgold, Boundary value problems of mathematical physics, vol. 2, Macmillan Co., New York, 1968, pp. 209-210 